\title{
Fetal abdominal tumors and cysts
}

\author{
Darrell L. Cass^ \\ Cleveland Clinic, Cleveland, OH, USA \\ Correspondence to: Darrell L. Cass, MD. Director of Fetal Surgery; Director, Fetal Care Center, Cleveland Clinic, 9500 Euclid Ave, A120, Cleveland, \\ OH 44122, USA. Email: cassd@ccf.org.
}

\begin{abstract}
This article reviews the contemporary diagnosis and management of the most common abdominal neoplasms and cystic lesions diagnosed in the fetus. Fetal tumors discussed include teratomas (sacrococcygeal, cervical or mediastinal), mesoblastic nephroma, nephroblastoma (Wilms' tumor), neuroblastoma, and hepatoblastoma. Fetal abdominal cystic lesions discussed include ovarian cyst, choledochal cyst, intestinal duplication cyst, mesenteric cyst, simple hepatic cyst, and meconium pseudocyst. We discuss the rare indications for fetal intervention or fetal surgery and other perinatal management, including prenatal interventions and fetal surgery for sacrococcygeal teratoma. The lesions reviewed are detected by widespread use of screening ultrasonography during pregnancy. Work-up for these abnormalities may include fetal MRI which enhances the diagnostic accuracy of abdominal tumors and cystic lesions and can aid in characterization of the lesion in relationship to surrounding anatomic structures. Accurate prenatal diagnosis of such lesions permits recommendations for optimal location and timing of delivery, and inclusion of appropriate caregivers and expertise to facilitate postnatal management. Perinatal management of the fetus with a neoplasm requires consideration of the optimal timing and mode of delivery, and pediatric oncology and surgical specialty care. The majority of tumors diagnosed antenatally have good prognosis with current multimodality treatment.
\end{abstract}

Keywords: Fetal surgery; sacrococcygeal teratoma; prenatal diagnosis; fetal ovarian cyst; perinatal neuroblastoma

Submitted Oct 30, 2020. Accepted for publication Apr 16, 2021.

doi: $10.21037 /$ tp-20-440

View this article at: http://dx.doi.org/10.21037/tp-20-440

\section{Introduction}

The title "fetal abdominal tumors and cysts" encompasses a broad array of neoplasms and malformations that might be diagnosed in the fetus, but for the purposes of this discussion we focus specifically on the most commonly diagnosed fetal abdominal neoplasms which include teratomas, congenital mesoblastic nephroma, neuroblastoma, and hepatoblastoma, and abdominal cystic lesions. Overall, these pathologies are rare. This review highlights the current approaches to prenatal imaging, differential diagnosis, antenatal natural history, and available treatment options for these most common conditions. We discuss the rare indications for fetal intervention or fetal surgery for specific lesions.

\section{Tumors}

Congenital tumors are very rare, with a reported incidence of between 2 and 14 infants per 100,000 live births, though there are likely many unreported cases as well (1). Approximately 130 malignant solid tumors are diagnosed in newborns in the United States each year. Most are diagnosed antenatally due to routine use of fetal ultrasonography (2).

Teratomas are the most common congenital tumors. Most involve the coccyx and sacrum [sacrococcygeal teratomas (SCT)], though teratomas may arise in the neck (cervical teratoma), brain, mediastinum, pericardium, and retroperitoneum along the midline. Other neoplasms

\footnotetext{
^ ORCID: 0000-0001-9082-2494.
} 
diagnosed in the fetus include the renal tumors, mesoblastic nephroma or, less commonly Wilms' tumor, neuroblastoma, and hepatoblastoma, though the incidence of these is much lower (3).

\section{Teratomas}

A teratoma, a neoplasm composed of all three germ layers, arises from totipotent somatic stem cells, thus this tumor may occur in any location where these cells are present. SCT, originating in Henson's node, are most common by far. In fact, SCT is the most common tumor of the fetus and newborn overall, with an incidence of 1 in 23,000 to 1 in 40,000 livebirths $(4,5)$. These tumors are 4 times more likely to occur in girls. Most SCT are benign, though the incidence of malignancy seems to be about 2-fold higher in those dying in utero or undergoing fetal resection compared to those removed postnatally, perhaps reflecting faster growing more vascular lesions in those examined prenatally (6). Epignathus is a rare form of oropharyngeal teratoma that arises from Rathke's pouch in the sphenoid region.

SCT has been classified traditionally by Altman and others by the relative amounts of presacral and external tumor present (7). Type I lesions are completely external, with no presacral components, whereas type IV lesions are completely internal (pelvic or abdominal) with no external components. Type II lesions have an external component that is larger than the internal pelvic component, whereas type III lesions extend further into the abdomen with an internal component that exceeds the external.

SCTs are diagnosed prenatally by ultrasound, though fetal MRI is helpful at assessing the exact anatomic location of the lesion relative to pelvic and abdominal structures. In some instances, these lesions are predominantly cystic with little vascularity evident by color flow Doppler, and in others these lesions may be largely solid and vascular, being supplied by branches off an enlarged middle sacral artery. Elevated combined cardiac output by fetal echocardiography is common; a condition that is associated with polyhydramnios and may progress to high-output cardiac failure (8). Fetal anemia secondary to tumor hemorrhage may contribute to the elevated cardiac outputs. Elevated fetal right heart pressures may lead to absent or reversed flow in the ductus venosus, worsened tricuspid valvular regurgitation, placental edema, placentomegaly, and maternal mirror syndrome. In fetuses with SCT, or any other vascular tumor, fetal hydrops is a marker of fetal right heart failure and a harbinger of impending fetal demise $(5,8)$.
The natural history of antenatally diagnosed SCT is poor compared to those presenting at birth. While the mortality rate for a newborn with SCT is less than $5 \%$, the mortality rate for fetal SCT is about $50 \%$ (9). Polyhydramnios is present in most cases of prenatally diagnosed SCT and likely results from renal hyperfiltration associated with the high output state. Polyhydramnios predisposes the pregnancy to preterm rupture of membranes and preterm delivery. Large pelvic elements from these tumors may compress other pelvic structures and lead to fetal bladder or bowel compression and disruption in extreme cases (10).

Fetuses with SCT should be followed closely with frequent fetal ultrasound to monitor tumor growth, amniotic fluid levels, and early signs of hydrops (11). For those fetuses with large, vascular "high risk" lesions, serial echocardiographic evaluations should be performed to evaluate cardiac function. Signs of high-output cardiac state include dilation of the inferior vena cava, increased flow velocity in the descending aorta, and increased combined ventricular output $(>550 \mathrm{~mL} / \mathrm{kg} / \mathrm{min})(12)$. There are a number of prognostic indicators that have been evaluated to predict outcomes for fetuses with SCT, including tumor size $>10 \mathrm{~cm}$, tumor rupture, the fraction of solid tumor component, increased vascularity, tumor volume growth rate higher than $150 \mathrm{~cm}^{3} /$ week, and prematurity (12-14). Tumor volume-to-fetal weight ratio (TFR) may also be a useful prognostic tool. Fetuses with a ratio greater than 0.12 by 24 weeks' gestation have been found to have a poor outcome compared to those with a lower ratio $(5,13)$. Though these prognostic markers may be useful, the management of a fetus needs to be guided by the specific sonographic and echocardiographic findings of each individual fetus.

Interventions aimed at preventing the high perinatal mortality associated with fetal SCT has been the focus of several centers. Investigators at the Children's Hospital of Philadelphia have advocated close follow-up and early delivery at 27 to 32 weeks. EXIT-to-resection has been used in some instances $(15,16)$. For a fetus with early signs of hydrops or placentomegaly at less than 27 weeks' gestation, resection in utero remains an option, though there is limited published data about these outcomes (1). The author of this review and colleagues while at Texas Children's Hospital performed open fetal surgical resection of two fetuses with giant vascular SCT with signs of evolving heart failure and hydrops at 25 (Figure 1) and 23 weeks' gestation (8). In both instances, the fetuses recovered well with resolution of hydrops, normalization of cardiac function, and delivery 

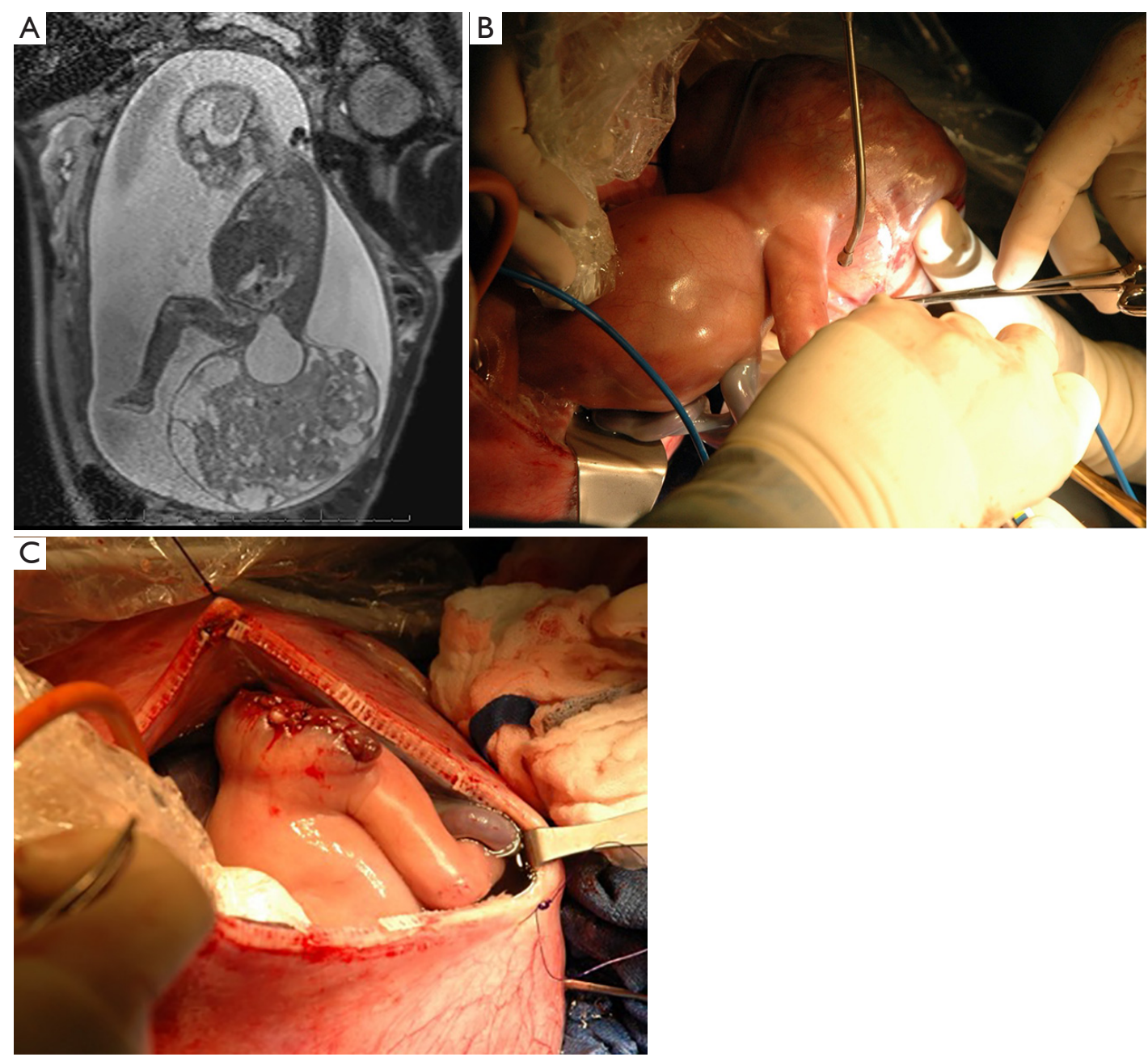

Figure 1 Fetal surgical resection of a 25-week female fetus with large vascular sacrococcygeal teratoma with high output heart failure and early fetal hydrops. (A) Fetal MRI shows large tumor with cystic and solid elements; (B) intraoperative photo of large fetal tumor; (C) intraoperative photo showing completed debulking of external aspect of tumor with ligation of blood supply to and from tumor and quickly closed skin; some cystic, non-vascular lesion remained within the fetal pelvis.

via cesarean section at 35 weeks' gestation. Both children recovered fully and are considered developmentally normal by their families at 12 and 4 years of age. Several investigators have advocated for use of radio-frequency thermal ablation (RFA), serial laser photocoagulation, thermocoagulation, or embolization using a coil or alcohol, in the fetal management of large, vascular SCT (17-19). These interventions performed percutaneously with ultrasound guidance are less invasive and avoid hysterotomy, but the specific indication for these interventions are often incomplete and published outcomes are poor. While coagulation of arterial inflow to the mass may be possible, it is likely more difficult to coagulate the complete venous and lymphatic drainage of the tumor. Tumor ischemia and necrosis may release intracellular potassium or reactive cytokines into the circulation of an already sick fetus which may cause fetal decompensation or early delivery. Furthermore, hemorrhage from friable tumor vessels has led to fetal demise, and thermal damage to surrounding structures including the anus and hip has led to permanent disability. In a review from investigators in Sao Paulo and Strasbourg who performed laser photocoagulation or sclerotherapy (intratumor alcohol injection) on 13 fetuses with SCT deemed "high risk", the median gestational age of delivery was 24.1 weeks and overall survival was $23.1 \%$ (18). In another series from Toronto, 5 fetuses with large SCT 
and high risk findings (though none with frank hydrops) underwent laser, coiling or RFA (17). Two died during the procedure and one died a day later. The two that survived were born 1-2 weeks following the interventions, likely due to poor ongoing fetal and placental physiology. In contrast, the post-procedure delivery interval following our open fetal surgery cases was 10 and 12 weeks with complete resolution of the fetal physiologic derangements. It is possible there is a learning curve to these less invasive approaches. While these therapies avoid maternal and uterine incisions, their benefit remains unproven and families should be counseled about the risks and outcomes from all therapeutic options.

Neonatal management of sacrococcygeal teratoma requires excision of the tumor. In general, complete excision of the tumor is sufficient therapy even for those tumors containing foci of yolk sac or immature elements. Chemotherapy is generally used as rescue therapy for those with recurrence or metastasis. Current survival rates following a complete excision approach $100 \%$ even for those with malignant disease (20).

\section{Renals tumors: mesoblastic nephroma and Wilm's}

Congenital renal tumors are rare, with mesoblastic nephroma being the most common to present in the first few months of life, and accounting for half of all neonatal renal masses (21). Ninety percent of mesoblastic nephromas present in the first year of life. These tumors are composed of mesenchymal tissue in the form of interlacing bundles of ovoid spindle-shaped cells. The "classical" form is most common, but a cellular variant is recognized in an increasing number of cases. The cellular variant has worse prognosis and shows a gene rearrangement with an ETV6 sequence fusion to NTRK3 (chromosome 12p13 and $15 \mathrm{q} 25$, respectively) (21).

Mesoblastic nephroma has been diagnosed rarely in the fetus (21-23). The differential diagnosis of a renal mass in the fetus includes Wilm's tumor, in addition to hydronephrosis and multicystic dysplastic kidney, focal renal dysplasia and diffuse nephroblastomatosis. Mesoblastic nephroma usually presents as a large unilateral renal mass $(4-8 \mathrm{~cm})$ that may appear nodular or homogeneous (24). These tumors are predominantly solid, but cystic areas are occasionally seen, usually due to hemorrhage with subsequent cystic degeneration. Polyhydramnios is a common associated finding in fetuses with mesoblastic nephroma, which may be due to compression on adjacent bowel, or increased fetal urine output from increased renal blood flow or impaired renal concentrating ability (25). Preterm rupture of membranes and preterm labor are common in these patients, and many deliver before 34 weeks' gestation. Hydrops has been described in association with these tumors which might be due to hemorrhage and anemia, increased vascularity and arteriovenous shunting, or compression from the mass on the portal vein or inferior vena cava. For fetuses with a renal mass, serial ultrasound is appropriate to monitor for complications with plans for delivery at a location with immediate access to neonatal surgical care. Fetal MRI aids in the accurate prenatal diagnosis of renal masses and can help in distinguishing mesoblastic nephroma from Wilms' tumor which tends to have a well-defined pseudocapsule (22). Bilateral renal lesions are more suggestive of Wilms' tumor rather than congenital mesoblastic nephroma.

In a multicenter review from France, about half of newborns with mesoblastic nephroma delivered before 34 weeks of gestation (23). In this study, $25 \%$ of fetuses had fetal distress that led to Cesarean section delivery, and $22 \%$ of neonates had hypertension due to renin secretion from the tumor or from native kidney (mass effects from the tumor leading to altered renal perfusion to normal kidney). Infants with mesoblastic nephroma may also show hypercalcemia which results from a paraneoplastic syndrome in which the tumor secretes parathyroid hormone-like peptides or prostaglandins.

In older children the cellular variant of mesoblastic nephroma is associated with a higher rate of local recurrence or distant metastases compared to those with typical histology, but that does not seem to be the case for fetuses or infants younger than 3 months of age (22).

Nephroblastoma (Wilms' tumor) accounts for $80 \%$ of renal neoplasms in children overall, while other tumor types, such as anaplastic sarcoma, clear cell sarcoma, rhabdoid tumor, and renal cell carcinoma account for the rest. The peak incidence of Wilms' tumor is at 2-3 years of age, though several cases have been described prenatally (21-23,25). Wilms' tumor is associated with many genetic conditions and syndromes, including Beckwith-Wiedemann, Denys-Drash, Klippel-Trenaunay, neurofibromatosis, and the WAGR complex.

For children with Wilms' tumor, 50\% will have hypertension $(21,22)$. Staging work-up includes chest $\mathrm{X}$-ray, abdominal CT scanning and abdominal ultrasound to look for tumor thrombus within the renal vein and inferior vena cava. A newborn with suspected Wilms' tumor should undergo exploratory laparotomy for radical nephroureterectomy and exploration of the contralateral 
kidney to exclude synchronous bilateral lesions. In cases of stage I Wilms' with tumors less than $550 \mathrm{gm}$ in patients younger than 2 years of age, adjuvant chemotherapy may be avoided, unlike in those with larger tumors or older age. Expected long-term survival rates for those with localized cancers or metastatic disease are greater than $90 \%$ and approximately $70 \%$, respectively.

\section{Neuroblastoma}

Neuroblastoma, one of the most common tumors of childhood, has an incidence of 1 in 10,000-30,000 children $(26,27)$. Neuroblastoma may be diagnosed in the third trimester of pregnancy. Prenatally diagnosed tumors tend to be small; 90\% involving the adrenal gland. Cystic and solid areas are typical and can be associated with hemorrhage and necrosis. Lesions that are mostly cystic have more favorable prognosis. Neuroblastoma may have microcalcifications with acoustic shadowing, encapsulation, and displacement of the kidney inferiorly. These tumors may arise in other areas along the sympathetic ganglia in the neck, chest, or intraabdominal paravertebral locations. Cervical lesions causing airway compression have been reported. A suprarenal mass associated with hepatomegaly is highly suggestive of the diagnosis of neuroblastoma.

Although most prenatally diagnosed neuroblastomas have a favorable prognosis, some tumors diagnosed in the perinatal period have more aggressive biologic behavior. Among 300 cases of fetal or perinatal neuroblastoma reported in 2012, $83 \%$ were stage I or II, but 2 had stage III or IV disease, and 5 had stage IVS disease (26). In addition, in a series by Jennings and colleagues, there were 14 stillbirths, 44 neonatal deaths, 2 late deaths, and only 10 survivors (28). Eight cases were associated with metastases to the placenta and one had umbilical cord metastases.

The newborn with suspected congenital neuroblastoma should have plain X-rays to look for calcifications, abdominal ultrasound, and CT scan or MRI to accurately stage the disease and define the exact tumor extent. Urine should be collected for the spot measurement of catecholamines and tumor metabolites, including homovanillic acid, vanillyl mandelic acid, dopamine, norepinephrine and epinephrine. Baseline liver enzymes should be obtained. Serum ferritin and neuron-specific enolase have prognostic significance in patients less than 1 year of age. Bone scan, MIBG scan and bone marrow aspiration and biopsy help complete preoperative staging. In cases of stage IVS disease, bluish, subcutaneous nodules from metastatic neuroblastoma may be seen on the infant's skin.

The treatment for newborns with neuroblastoma depends on the staging. Generally, neonatal neuroblastomas have a DNA index more than 1 , and less than $5 \%$ have $\mathrm{N}$-myc amplification, indicating a more favorable prognosis. Chemotherapy is indicated for those with stage IVS disease with $\mathrm{N}$-myc amplification or other unfavorable markers. Observation alone is appropriate for those without unfavorable findings. The overall long-term survival for prenatally diagnosed neuroblastoma is over $90 \%$ (26). There are no indications for prenatal intervention.

\section{Otber fetal tumors}

Tumors of the liver are rare in the fetus and newborn, accounting for about $5 \%$ of perinatal neoplasms. The most common primary liver lesion is hemangioma, followed by mesenchymal hamartoma and hepatoblastoma. However, metastatic lesions are more common than primary liver tumors. The most common tumor that metastasizes to the liver of the fetus or neonate is neuroblastoma, followed by leukemia, yolk-sac tumor from sacrococcygeal teratoma, and rhabdoid tumor of the kidney $(1,3)$.

Hepatoblastoma, the most common primary liver tumor occurring in the first year of life, can be detected in the fetus. Such tumors may be associated with other congenital anomalies and malformation syndromes, such as hemihypertrophy syndromes including BeckwithWiedemann syndrome and intestinal adenomatous polyposis. On prenatal ultrasound, hepatoblastoma are commonly echogenic, solid and show calcifications. In contrast, hamartomas typically appear as an irregular cyst sonographically, and are associated with both oligohydramnios and polyhydramnios.

The evaluation of a fetus with hepatic tumor includes fetal ultrasound, fetal MRI and echocardiography to look for signs of elevated cardiac output. Ideally these babies should be delivered at a center with immediate access to neonatal oncology and surgical care. Postnatally, evaluation and treatment includes, measurement of serum alpha fetoprotein, further imaging, and surgical resection if possible. Those deemed unresectable are treated with biopsy and neoadjuvant chemotherapy with definitive resection following several cycles.

\section{Fetal abdominal cysts}

The prenatal diagnosis of abdominal cystic lesions is 
relatively common. The differential diagnosis of a fetal cystic abdominal mass is extensive and includes: ovarian cyst (female fetuses), choledochal cyst, hepatic, splenic or pancreatic cysts, mesenteric cysts, meconium pseudocyst, adrenal hemorrhagic cysts, renal cysts, and intestinal duplication cysts (29-31). Other anomalies in the differential may include intraabdominal extralobar bronchopulmonary sequestration with cystic changes, and some cystic tumors, such as neuroblastoma, but these are even rarer. Generally, the more precise diagnosis can be narrowed by relying upon anatomic relationships and associated findings such as the so-called bowel wall signature seen in duplication cysts (32), calcifications seen in meconium pseudocysts, the location to the side of the bladder in ovarian cysts, the relationship to the hepatic artery in choledochal cysts, or the surrounding parenchyma and location as seen in fetuses with hepatic, splenic, pancreatic, or adrenal cysts.

An intraabdominal cyst is most frequently detected in the second or third trimester. Isolated cysts seen earlier in gestation are usually associated with good outcome (33). During the development of intra-abdominal structures, cysts may form mainly from either the gastrointestinal or genitourinary tract. The cysts can resolve spontaneously. Though we may suspect the pathology, it remains difficult to be definitive in the fetus (30). For fetuses with abdominal cystic lesions, the correlation between prenatal and postnatal diagnosis ranges from $72.3 \%$ to $94.8 \%$ overall (34). Fetal MRI has been shown to enhance the fetal diagnosis of intra-abdominal cysts $(30,35)$. In a study by Hugele and colleagues from 2015, fetal MRI was shown to increase the diagnostic accuracy for fetuses with abdominal cystic lesions from $51 \%$ to $73.4 \%$; correcting the diagnosis or providing more precise information in $26.5 \%$ of cases (36). Specific prenatal diagnosis may help in counseling and planning delivery in a setting with immediate access to appropriate specialists, including neonatology, pediatric general surgery, pediatric hepatology, nephrology or urology.

When comparing male to female fetuses, cysts in males are diagnosed at an earlier gestation and are associated with a higher rate of associated anomalies (37). Fetuses with anorectal anomalies can be seen to have a "pelvic cyst" early in gestation, likely representing a dilated distal rectum in those with atresias. The "cyst" may later resolve due to presumed decompression of the dilated rectum through a rectal fistula (bladder, urethral, vestibular, or perineal). Fetal MRI has been shown to be useful in examining the perianal muscle complex in these cases. Cysts in female fetuses are usually larger, since most are ovarian. When excluding ovarian cysts, the size of the cysts is comparable between female and male fetuses.

The presence of congenital anorectal anomalies is low overall (1:5,000 live births), however this anomaly was more common in those diagnosed with a fetal cystic abdominal lesion (37). In those confirmed to have anorectal anomalies at birth, the fetal cyst regressed in 1 of 3 females, and 2 of 3 males. Most fetuses with an anorectal anomaly have additional associated anomalies.

\section{Ovarian cyst}

Ovarian cysts arise from ovarian follicles. The cause of fetal ovarian cysts is not clear, but most likely results from stimulation of fetal ovaries by maternal estrogens, fetal gonadotropins, and placental chorionic gonadotropin. Fetal ovarian cysts are common in pregnancies complicated by maternal diabetes, rhesus isoimmunization, and pre-eclampsia. Bilateral cysts can be seen in $5 \%$ of fetal cases (38).

The incidence of ovary cysts is about 1 in 2,600 pregnancies. They are generally seen in the $2^{\text {nd }}$ or $3^{\text {rd }}$ trimester (example in Figure 2). Cysts are categorized as simple or complex based on the presence of internal echoes, septations, or intermixed echogenic foci. Complex cysts have a higher incidence of prenatal ovarian torsion. Ovarian cysts are considered physiologic when less than $2 \mathrm{~cm}$ in size; most of these regress (39). There is an increased risk of spontaneous ovarian torsion in cysts equal or greater than $4 \mathrm{~cm}$ (40). Also, larger cysts rarely may cause bowel obstruction and associated polyhydramnios (41-44). The differential diagnosis of fetal ovarian cyst includes mesenteric cyst, urachal cyst and enteric duplication cyst.

Fetal cyst aspiration has been described in the management of larger presumed ovarian cysts, though the benefits of this intervention have not been proven $(45,46)$. In cases where the cyst causes bowel or other organ compression leading to potential obstruction and polyhydramnios the rationale seems greater, but these are exceptional cases. The unknown potential benefit of preventing ovarian torsion needs to be weighed against the potential risks, which include premature rupture of membranes, injury to bowel or associated structures, hemorrhage leading to later adhesions and obstruction, infection, or aspiration of a lesion that is not an ovarian cyst.

The treatment of a neonate with ovarian cyst is not standardized. For asymptomatic girls, families are counseled about observation versus diagnostic laparoscopy. Operation 

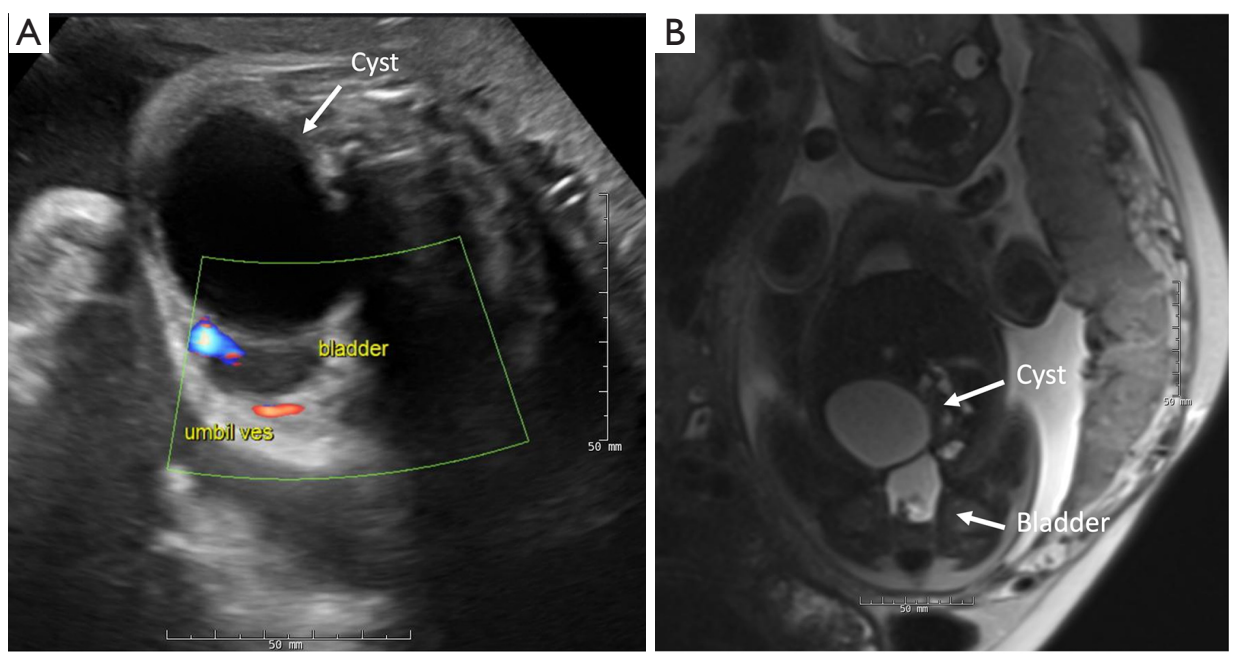

Figure 2 Fetal imaging of a female fetus with right ovarian cyst. (A) Fetal ultrasound images at $331 / 7$ weeks gestation, cyst $5.2 \mathrm{~cm}$; (B) fetal MRI images from $342 / 7$ weeks gestation, cyst $5.3 \mathrm{~cm}$. Prenatally, ovarian cyst was suspected.
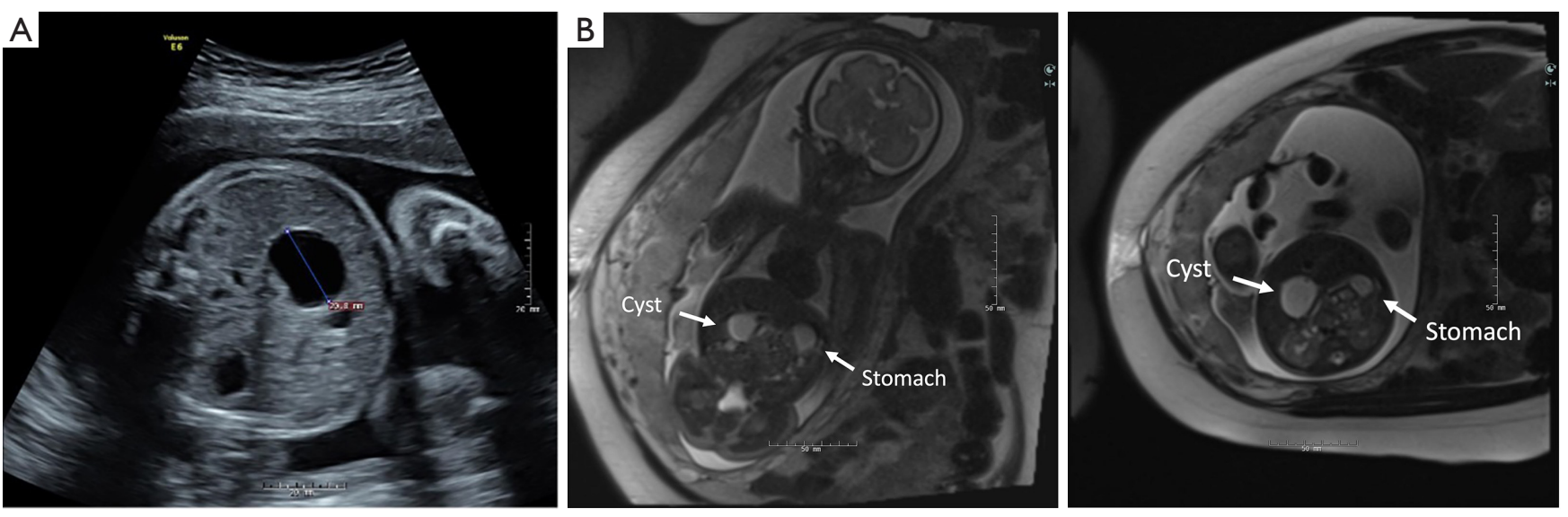

Figure 3 Fetal imaging of a female fetus with choledochal cyst. (A) Fetal ultrasound at $245 / 7$ weeks, cyst 2 cm; (B) fetal MRI at 27 6/7 weeks, cyst at porta hepatis, $2.4 \mathrm{~cm}$. A prenatal diagnosis of choledochal cyst was made.

may be favored for those with larger cysts $(>4 \mathrm{~cm})$ or complex cysts. Theoretically, ovarian torsion may occur during periods of observation. Similarly, a necrotic torsed ovary has been reported to cause bowel obstruction and urinary tract obstruction in infants. Case series in which early operation was associated with high rates of adnexal preservation support the concept of early laparoscopic exploration. However, in a review from Brandt and colleagues, ultrasound evidence of torsion at or before birth was found in $92 \%$ of patients with surgical cases, suggesting that most torsion occurs prenatally and the risk of postnatal torsion may be low (44).

\section{Choledochal cyst}

Choledochal cysts are rare congenital cystic dilations of the biliary tree and are classified by the portion of the biliary tree affected (47). The most common form consists of fusiform dilation of the common bile duct: type I, accounting for $85-90 \%$ of cases. Nearly all cases diagnosed prenatally are of this type (example in Figure 3). Other forms are a diverticulum of the common bile duct (type II), an intraduodenal or intrapancreatic choledochoceal (type III), and a form that has multiple extrahepatic cysts with or without intrahepatic cysts (type IV). Caroli's disease consists of single or multiple intrahepatic cysts associated 
with hepatic fibrosis and a normal extrahepatic biliary tree (type V). Choledochal cyst is a rare anomaly with an estimated occurrence ranging from 1 case in 100,000 to 150,000 livebirths to 1 case in 2 million livebirths. It is more common in females (2.5:1) and in Asian populations (31).

The wall of a choledochal cyst is usually thickened with dense connective tissue interlaced with strands of smooth-muscle fibers associated with some inflammatory reaction. Many prenatally diagnosed choledochal cysts have extrahepatic biliary obstruction (48). Theories regarding the cause of choledochal cyst relate to congenital weakness of the wall of the bile ducts combined with distal obstruction. Todani has noted that many patients with choledochal cyst have an anomalous arrangement of the pancreatic and biliary duct system in which the pancreatic duct enters at an abnormal angle and more proximal on the duct permitting reflux of pancreatic enzymes upward into the common bile duct, potentially causing damage to the common bile duct wall in utero. Though this may be the cause in some cases, a "long common channel" has been noted in up to $50 \%$ of patients with biliary disorders other than choledochal cyst. Furthermore, choledochal cysts have been diagnosed as early as 15 weeks of gestation, well before the development of pancreatic exocrine function.

The differential diagnosis of a cystic mass in the right upper quadrant includes hepatic or omental cysts, adrenal cysts, renal cysts, a hydronephrotic renal pelvis in the right kidney, a dilated loop of bowel, mesenteric cysts, duodenal duplications, gallbladder duplications, simple hepatic cysts, duodenal atresia and situs inversus. In females, ovarian cysts can move up into the right upper quadrant. Color Doppler studies that demonstrate an intimate association of the cyst with the hepatic artery and portal vein suggest a biliary origin.

Choledochal cysts can be associated with biliary obstruction. Hepatic fibrosis and bile ductule proliferation has been seen in these patients. Cystic biliary atresia can be differentiated from choledochal cyst by the presence of rising conjugated hyperbilirubinemia, bile ductule proliferation, and hepatic fibrosis progressing to cirrhosis. Infants with signs of biliary obstruction should undergo operation promptly. For infants who are asymptomatic with normal eating, bowel movements and growth, and normal laboratory values, the timing of operation is less clearly established. Recent studies that demonstrate a high incidence of hepatic fibrosis even in asymptomatic children with choledochal cysts, support the concept of earlier surgery rather than later (49). Surgical treatment involves complete excision of the cyst with either Roux-en-Y hepaticojejunostomy or hepaticoduodenostomy (becoming more common) $(50,51)$.

\section{Hepatic cysts}

Hepatic cysts are most often isolated, simple cysts that are thought to arise from aberrant bile ducts or intrahepatic, peribiliary glands. These simple cysts are more common in girls and do not communicate with the normal biliary system (52). Many of these cysts detected prenatally shrink or resolve postnatally; rarely they may grow and cause symptoms postnatally (53). In rare cases, hepatic cysts can grow to fill the entire peritoneal cavity. This finding can be distinguished from ascites by its compression of the bowel into the retroperitoneum behind the cyst. There are case reports of fetal cyst aspiration, though these large cysts usually require no treatment.

Differentiating a cyst as hepatic, splenic, mesenteric, pancreatic, or gastric or duodenal duplication cyst can be difficult unless the cyst is clearly surrounded by distinguishable organ parenchyma. Fetal MRI can be helpful in enhancing the specificity of fetal diagnosis for these lesions (54). Charlesworth reviewed a series of 15 cases of hepatic cysts of which 9 had serial ultrasound scans. In 5 the cyst enlarged, in 3 there was no change, and 1 case had a decrease in cyst size (55).

\section{Splenic cysts}

Splenic cysts are usually simple serous epithelial-lined cysts in the upper pole of the spleen. These cysts are often diagnosed prenatally in the third trimester and may be difficult to distinguish from adrenal or pancreatic cysts (56).

\section{Pancreatic cysts}

Pancreatic cysts arise from a developmental anomaly of the pancreatic ductal system usually in the body or tail of the pancreas. These cysts are epithelial lined and are not pseudocysts. These may occur as part of BeckwithWiedemann syndrome, polycystic disease of the pancreas and kidney, or Hippel-Lindau disease but are usually multiple in these cases (57).

\section{Duplication cysts}

Intestinal duplication cysts can occur at any point along the 

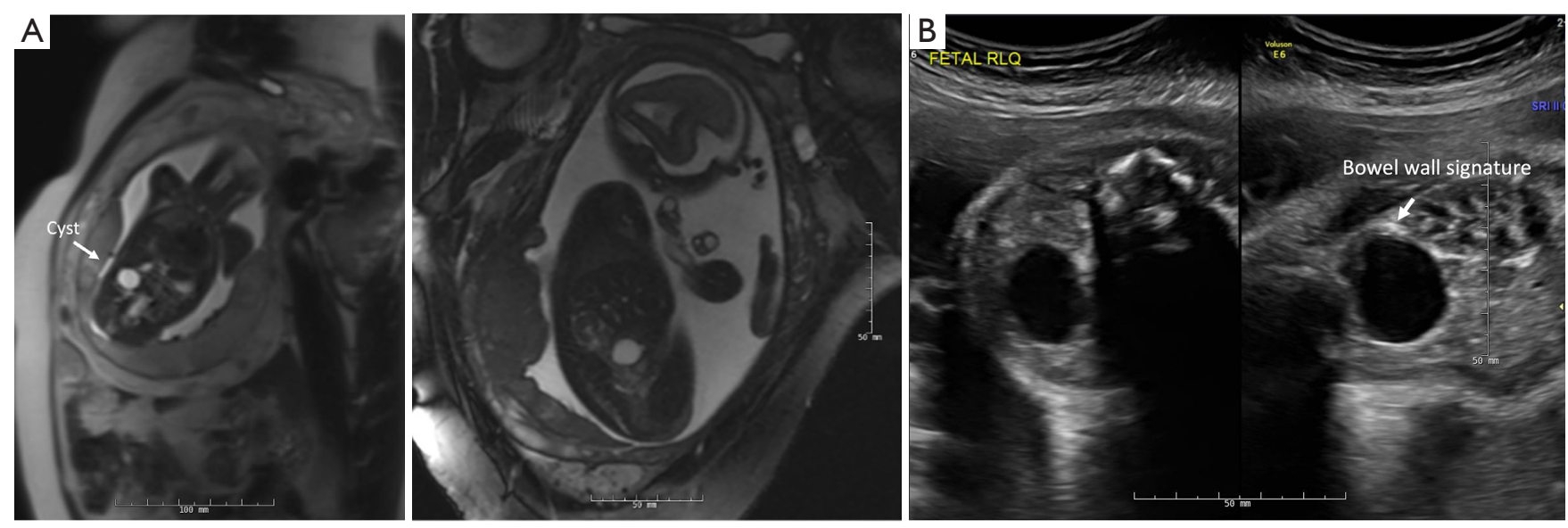

Figure 4 Fetal imaging of a female fetus with ileal duplication cyst. (A) Fetal MRI at 24 weeks gestation, cyst $1.5 \mathrm{~cm}$; (B) fetal ultrasound at 36 6/7 weeks; cyst $2.4 \mathrm{~cm}$ and displays "bowel wall signature" (32). Prenatally, intestinal duplication cyst was suspected.

gastrointestinal tract, though they are most common in the jejunum and ileum (53\%), and may occur in the colon (18\%), duodenum (6\%) or stomach (4\%) (example is in Figure 4). Colonic duplications may be associated with duplications of the genitourinary system. Intestinal duplication may occur in association with vertebral and spinal cord anomalies such as hemivertebrae, anterior myelomeningocele, and spinal cord duplications $(29,58,59)$.

Enteric duplication cysts may present with findings suggestive of bowel obstruction with dilated proximal loops of bowel. Polyhydramnios has been seen for fetuses with more proximal duplication cysts of the stomach, pylorus, duodenum or jejunum.

\section{Mesenteric cysts}

Mesenteric cysts are cystic lymphangiomas (lymphatic malformations) that appear unilocular or multilocular arising in the mesentery of the bowel (60). These lesions may be associated with genetic syndromes such as Turner, Noonan, Fryns or trisomy 18 and 21.

\section{Meconium pseudocyst}

Meconium pseudocyst is a manifestation of meconium peritonitis in which there is a fetal intestinal perforation (small intestine or colon) with leakage of fetal intestinal contents that become contained in an inflammatory, cystic space. Extraluminal abdominal calcifications are present in $85 \%$ of cases (61). Fetal ascites, bowel dilation, and polyhydramnios may be associated findings (62).
Interestingly, these prenatal findings may resolve and the intestinal perforation can heal without forming an intestinal atresia or stenosis. Prenatally diagnosed meconium peritonitis differs from postnatally diagnosed cases in reduced morbidity, lower incidence of cystic fibrosis, and an overall better prognosis. The better prognosis may be selection bias since prenatal ultrasound is more sensitive in detecting calcifications compared to postnatal X-rays thus diagnosing more mild cases, compared to those that are detected postnatally. Also, many fetal cases may heal, leading to favorable clinical status at birth. Prenatal meconium pseudocyst has presented as meconium per vagina in a newborn girl (63).

\section{Prenatal management of the fetus with abdominal cyst}

The fetus noted to have to have a cystic abdominal lesion should undergo a detailed ultrasound in an effort to define the cyst, but also to screen for possible associated anomalies. If there is a single umbilical artery or other markers of aneuploidy, a genetics consultation and amniocentesis may be appropriate. A fetal MRI may be complementary in characterizing the etiology of the cystic lesion. The pregnancy should be followed by serial prenatal ultrasound due to risk for bowel obstruction, polyhydramnios and preterm labor. Consultation with neonatology and pediatric surgery will be useful and may help to define the best delivery location and possible need for immediate access to neonatal surgical care.

Fetal abdominal cysts do not typically benefit from fetal intervention. Rare exceptions may be very large 
ovarian or hepatic cysts with associated compression on adjacent organs, and bowel obstruction that may lead to polyhydramnios.

\section{Postnatal management of the fetus with abdominal cyst}

The most important aspect in the management of a newborn with a cystic abdominal lesion is an accurate diagnosis and exclusion of associated conditions. Physical exam should include an evaluation for dysmorphic features, with genetics consultation as appropriate. The timing of the work-up will depend on the presence of symptoms (prenatal bowel dilation, compression on surrounding structures) and the suspected diagnosis. Plain $\mathrm{X}$-rays and abdominal ultrasound are the most common initial studies. CT scan, MRI, and hepatobiliary nuclear medicine studies may be adjuncts, particularly for hepato-pancreatic lesions. Surgical excision is appropriate for many of these lesions, though the exact timing may vary. Duplication cysts should likely be excised electively due to risk of further growth, bowel obstruction and intestinal bleeding. Choledochal cysts may be associated with biliary atresia type abnormalities and fibrotic changes in the liver that will benefit from earlier excision to avoid the risk for progressive liver damage.

\section{Acknowledgments}

The author would like to acknowledge the mothers and families that place their trust in us to provide the very best care for their children, both before and after birth.

Funding: None.

\section{Footnote}

Provenance and Peer Review: This article was commissioned by the Guest Editors (Eric B. Jelin and George B. Mychaliska) for the series "Fetal Surgery" published in Translational Pediatrics. The article has undergone external peer review.

Conflicts of Interest: The author has completed the ICMJE uniform disclosure form (available at http://dx.doi. org/10.21037/tp-20-440). The series "Fetal Surgery" was commissioned by the editorial office without any funding or sponsorship. The author has no other conflicts of interest to declare.

Ethical Statement: The author is accountable for all aspects of the work in ensuring that questions related to the accuracy or integrity of any part of the work are appropriately investigated and resolved.

Open Access Statement: This is an Open Access article distributed in accordance with the Creative Commons Attribution-NonCommercial-NoDerivs 4.0 International License (CC BY-NC-ND 4.0), which permits the noncommercial replication and distribution of the article with the strict proviso that no changes or edits are made and the original work is properly cited (including links to both the formal publication through the relevant DOI and the license). See: https://creativecommons.org/licenses/by-nc-nd/4.0/.

\section{References}

1. Peiro JL, Sbragia L, Scorletti F, et al. Perinatal Management of Fetal Tumors. Curr Pediatr Rev 2015;11:151-63.

2. Bader JL, Miller RW. US cancer incidence and mortality in the first year of life. Am J Dis Child 1979;133:157-9.

3. Bruny J, Crombleholme TM. Perinatal management of infant tumors and the promise of fetal surgery. Curr Opin Pediatr 2013;25:31-9.

4. Forrester MB, Merz RD. Descriptive epidemiology of teratoma in infants, Hawaii, 1986-2001. Paediatr Perinat Epidemiol 2006;20:54-8.

5. Rodriguez MA, Cass DL, Lazar DA, et al. Tumor volume to fetal weight ratio as an early prognostic classification for fetal sacrococcygeal teratoma. J Pediatr Surg 2011;46:1182-5.

6. Sebire NJ, Fowler D, Ramsay AD. Sacrococcygeal tumors in infancy and childhood; a retrospective histopathological review of 85 cases. Fetal Pediatr Pathol 2004;23:295-303.

7. Altman RP, Randolph JG, Lilly JR. Sacrococcygeal teratoma: American Academy of Pediatrics Surgical Section Survey-1973. J Pediatr Surg 1974;9:389-98.

8. Cass DL, Olutoye OO, Ayres NA, et al. Defining hydrops and indications for open fetal surgery for fetuses with lung masses and vascular tumors. J Pediatr Surg 2012;47:40-5.

9. Bond SJ, Harrison MR, Schmidt KG, et al. Death due to high-output cardiac failure in fetal sacrococcygeal teratoma. J Pediatr Surg 1990;25:1287-91.

10. Partridge EA, Canning D, Long C, et al. Urologic and anorectal complications of sacrococcygeal teratomas: prenatal and postnatal predictors. J Pediatr Surg 2014;49:139-42; discussion 142-3.

11. Wohlmuth C, Bergh E, Bell C, et al. Clinical Monitoring 
of Sacrococcygeal Teratoma. Fetal Diagn Ther 2019;46:333-40.

12. Shue E, Bolouri M, Jelin EB, et al. Tumor metrics and morphology predict poor prognosis in prenatally diagnosed sacrococcygeal teratoma: a 25-year experience at a single institution. J Pediatr Surg 2013;48:1225-31.

13. Akinkuotu AC, Coleman A, Shue E, et al. Predictors of poor prognosis in prenatally diagnosed sacrococcygeal teratoma: A multiinstitutional review. J Pediatr Surg 2015;50:771-4.

14. Byrne FA, Lee H, Kipps AK, et al. Echocardiographic risk stratification of fetuses with sacrococcygeal teratoma and twin-reversed arterial perfusion. Fetal Diagn Ther 2011;30:280-8.

15. Roybal JL, Moldenhauer JS, Khalek N, et al. Early delivery as an alternative management strategy for selected high-risk fetal sacrococcygeal teratomas. J Pediatr Surg 2011;46:1325-32.

16. Baumgarten HD, Gebb JS, Khalek N, et al. Preemptive Delivery and Immediate Resection for Fetuses with High-Risk Sacrococcygeal Teratomas. Fetal Diagn Ther 2019;45:137-44.

17. Van Mieghem T, Al-Ibrahim A, Deprest J, et al. Minimally invasive therapy for fetal sacrococcygeal teratoma: case series and systematic review of the literature. Ultrasound Obstet Gynecol 2014;43:611-9.

18. Sananes N, Javadian P, Schwach Werneck Britto I, et al. Technical aspects and effectiveness of percutaneous fetal therapies for large sacrococcygeal teratomas: cohort study and literature review. Ultrasound Obstet Gynecol 2016;47:712-9.

19. Litwinska M, Litwinska E, Janiak K, et al. Percutaneous Intratumor Laser Ablation for Fetal Sacrococcygeal Teratoma. Fetal Diagn Ther 2020;47:138-44.

20. Peiro JL, Sbragia L, Scorletti F, et al. Management of fetal teratomas. Pediatr Surg Int 2016;32:635-47.

21. Glick RD, Hicks MJ, Nuchtern JG, et al. Renal tumors in infants less than 6 months of age. J Pediatr Surg 2004;39:522-5.

22. Berger M, von Schweinitz D. Current Management of Fetal and Neonatal Renal Tumors. Curr Pediatr Rev 2015;11:188-94.

23. Leclair MD, El-Ghoneimi A, Audry G, et al. The outcome of prenatally diagnosed renal tumors. J Urol 2005;173:186-9.

24. Miniati D, Gay AN, Parks KV, et al. Imaging accuracy and incidence of Wilms' and non-Wilms' renal tumors in children. J Pediatr Surg 2008;43:1301-7.
25. Bove KE. Wilms' tumor and related abnormalities in the fetus and newborn. Semin Perinatol 1999;23:310-8.

26. Nuchtern JG. Perinatal neuroblastoma. Semin Pediatr Surg 2006;15:10-6.

27. Newman EA, Nuchtern JG. Recent biologic and genetic advances in neuroblastoma: Implications for diagnostic, risk stratification, and treatment strategies. Semin Pediatr Surg 2016;25:257-64.

28. Jennings RW, LaQuaglia MP, Leong K, et al. Fetal neuroblastoma: prenatal diagnosis and natural history. J Pediatr Surg 1993;28:1168-74.

29. Lewis S, Walker J, McHoney M. Antenatally detected abdominal cyst: Does cyst size and nature determine postnatal symptoms and outcome? Early Hum Dev 2020;147:105102.

30. Lv M, Zhao B, Luo Q. Prenatal diagnosis and prognosis assessment of fetal intra-abdominal cystic lesions: a retrospective study in 264 cases. J Obstet Gynaecol 2019;39:922-7.

31. Sanna E, Loukogeorgakis S, Prior T, et al. Fetal abdominal cysts: antenatal course and postnatal outcomes. J Perinat Med 2019;47:418-21.

32. Di Serafino M, Mercogliano C, Vallone G. Ultrasound evaluation of the enteric duplication cyst: the gut signature. J Ultrasound 2016;19:131-3.

33. Khalil A, Cooke PC, Mantovani E, et al. Outcome of firsttrimester fetal abdominal cysts: cohort study and review of the literature. Ultrasound Obstet Gynecol 2014;43:413-9.

34. Marchitelli G, Stirnemann J, Acanfora MM, et al. Prenatal diagnosis of intra-abdominal cystic lesions by fetal ultrasonography: diagnostic agreement between prenatal and postnatal diagnosis. Prenat Diagn 2015;35:848-52.

35. Gupta P, Sharma R, Kumar S, et al. Role of MRI in fetal abdominal cystic masses detected on prenatal sonography. Arch Gynecol Obstet 2010;281:519-26.

36. Hugele F, Dumont C, Boulot P, et al. Does prenatal MRI enhance fetal diagnosis of intra-abdominal cysts? Prenat Diagn 2015;35:669-74.

37. Husen M, Schut PC, Neven ACH, et al. Differences in Origin and Outcome of Intra-Abdominal Cysts in Male and Female Fetuses. Fetal Diagn Ther 2019;46:166-74.

38. Heling KS, Chaoui R, Kirchmair F, et al. Fetal ovarian cysts: prenatal diagnosis, management and postnatal outcome. Ultrasound Obstet Gynecol 2002;20:47-50.

39. Tyraskis A, Bakalis S, Scala C, et al. A retrospective multicenter study of the natural history of fetal ovarian cysts. J Pediatr Surg 2018;53:2019-22.

40. Bascietto F, Liberati M, Marrone L, et al. Outcome of 
fetal ovarian cysts diagnosed on prenatal ultrasound examination: systematic review and meta-analysis. Ultrasound Obstet Gynecol 2017;50:20-31.

41. Cass DL. Ovarian torsion. Semin Pediatr Surg 2005;14:86-92.

42. Bornstein E, Barnhard Y, Ferber A, et al. Acute progression of a unilateral fetal ovarian cyst to complex bilateral cysts causing acute polyhydramnios. J Ultrasound Med 2006;25:523-6.

43. Comparetto C, Giudici S, Coccia ME, et al. Fetal and neonatal ovarian cysts: what's their real meaning? Clin Exp Obstet Gynecol 2005;32:123-5.

44. Brandt ML, Helmrath MA. Ovarian cysts in infants and children. Semin Pediatr Surg 2005;14:78-85.

45. Diguisto C, Winer N, Benoist G, et al. In-utero aspiration vs expectant management of anechoic fetal ovarian cysts: open randomized controlled trial. Ultrasound Obstet Gynecol 2018;52:159-64.

46. Tyraskis A, Bakalis S, David AL, et al. A systematic review and meta-analysis on fetal ovarian cysts: impact of size, appearance and prenatal aspiration. Prenat Diagn 2017;37:951-8.

47. Karrer FM, Hall RJ, Stewart BA, et al. Congenital biliary tract disease. Surg Clin North Am. 1990;70:1403-18.

48. Soares KC, Goldstein SD, Ghaseb MA, et al. Pediatric choledochal cysts: diagnosis and current management. Pediatr Surg Int 2017;33:637-50.

49. Diao M, Li L, Cheng W. Timing of surgery for prenatally diagnosed asymptomatic choledochal cysts: a prospective randomized study. J Pediatr Surg 2012;47:506-12.

50. Sun R, Zhao N, Zhao K, et al. Comparison of efficacy and safety of laparoscopic excision and open operation in children with choledochal cysts: A systematic review and update meta-analysis. PLoS One 2020;15:e0239857.

51. Diaz Ramos NM, Lugo-Vicente H. Choledochal Cyst: Hepaticoduodenostomy or hepaticojejunostomy? Bol Asoc Med P R 2016;108:41-6.

Cite this article as: Cass DL. Fetal abdominal tumors and cysts. Transl Pediatr 2021;10(5):1530-1541. doi: 10.21037/tp20-440
52. Otani Y, Takayasu H, Ishimaru Y, et al. Secretion and expression of epithelial markers supports the biliary origin of solitary nonparasitic cyst of the liver in infancy. J Pediatr Surg 2005;40:e27-30.

53. Rogers TN, Woodley H, Ramsden W, et al. Solitary liver cysts in children: not always so simple. J Pediatr Surg 2007;42:333-9.

54. Nordin AB, Fallon SC, Carter BA, et al. Congenital hepatic cyst with antenatal diagnosis: a case report and literature review. Pediatr Surg Int 2013;29:847-50.

55. Charlesworth P, Ade-Ajayi N, Davenport M. Natural history and long-term follow-up of antenatally detected liver cysts. J Pediatr Surg 2007;42:494-9.

56. Sauvageot C, Faure JM, Mousty E, et al. Prenatal and postnatal evolution of isolated fetal splenic cysts. Prenat Diagn 2018;38:390-4.

57. Warnock WT, Khoshnam N, Bird KM, et al. Congenital Cyst of the Pancreas: A Case Report and Review of Literature. Fetal Pediatr Pathol 2016;35:265-71.

58. Foley PT, Sithasanan N, McEwing R, et al. Enteric duplications presenting as antenatally detected abdominal cysts: is delayed resection appropriate? J Pediatr Surg 2003;38:1810-3.

59. Fahy AS, Pierro A. A Systematic Review of Prenatally Diagnosed Intra-abdominal Enteric Duplication Cysts. Eur J Pediatr Surg 2019;29:68-74.

60. Chang TS, Ricketts R, Abramowsky CR, et al. Mesenteric cystic masses: a series of 21 pediatric cases and review of the literature. Fetal Pediatr Pathol 2011;30:40-4.

61. Catania VD, Briganti V, Di Giacomo V, et al. Fetal intraabdominal cysts: accuracy and predictive value of prenatal ultrasound. J Matern Fetal Neonatal Med 2016;29:1691-9.

62. Kamata S, Nose K, Ishikawa S, et al. Meconium peritonitis in utero. Pediatr Surg Int 2000;16:377-9.

63. Gauderer MW, Cass DL. Meconium per vaginam secondary to ileal atresia and meconium peritonitis. Pediatric Surgery International 1992;7:64-6. 